\title{
MR Venous Flow in Sigmoid Sinus Diverticulum
}

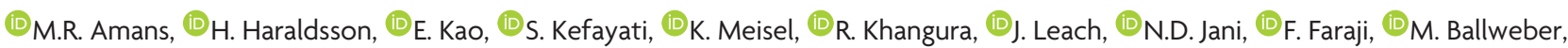 \\ (1)W. Smith, and $\mathbb{D}^{\mathrm{D}}$. Saloner
}

\begin{abstract}
BACKGROUND AND PURPOSE: Case reports demonstrate that coiling of a sigmoid sinus diverticulum can treat pulsatile tinnitus. We hypothesized that MR imaging 4D flow and computational fluid dynamics would reveal distinct blood flow patterns in the venous outflow tract in these patients.
\end{abstract}

MATERIALS AND METHODS: Patients with pulsatile tinnitus of suspected venous etiology underwent MR imaging at 3T, using venous phase contrast-enhanced MR angiography, 4D flow, and 2D phase contrast. The contrast-enhanced MRA contours were evaluated to determine the presence and extent of a sigmoid sinus diverticulum. Computational fluid dynamics analysis was performed using the 4D flow inlet flow and the luminal contours from contrast-enhanced MRA as boundary conditions. In addition, computational fluid dynamics was performed for the expected post treatment conditions by smoothing the venous geometry to exclude the sigmoid sinus diverticulum from the anatomic boundary conditions. Streamlines were generated from the 4D flow and computational fluid dynamics velocity maps, and flow patterns were examined for the presence of rotational components.

RESULTS: Twenty-five patients with pulsatile tinnitus of suspected venous etiology and 10 control subjects were enrolled. Five (20\%) of the symptomatic subjects had sigmoid sinus diverticula, all associated with an upstream stenosis. In each of these patients, but none of the controls, a stenosis-related flow jet was directed toward the opening of the sigmoid sinus diverticulum with rotational flow patterns in the sigmoid sinus diverticulum and parent sigmoid sinus on both 4D flow and computational fluid dynamics.

CONCLUSIONS: Consistent patterns of blood flow can be visualized in a sigmoid sinus diverticulum and the parent sinus using 4D flow and computational fluid dynamics. Strong components of rotational blood flow were seen in subjects with sigmoid sinus diverticula that were absent in controls.

ABBREVIATIONS: $C F D=$ computational fluid dynamics; $4 \mathrm{DF}=4 \mathrm{D}$ flow; $\mathrm{PT}=$ pulsatile tinnitus; $\mathrm{SSD}=$ sigmoid sinus diverticulum; $\mathrm{SSIJ}=$ sigmoid sinus and internal jugular vein; UCSF = University of California, San Francisco

T innitus is the auditory perception of sound in the absence of an extracorporeal source, which affects millions of Americans. ${ }^{1}$ Patients' lives can be severely impacted by tinnitus, and it is not uncommon for patients to have insomnia, depression, or even

Received March 27, 2018; accepted after revision August 20.

From the Departments of Radiology and Biomedical Imaging (M.R.A., H.H., E.K., S.K., R.K., J.L., N.D.J., F.F., M.B., D.S.) and Neurology (K.M., W.S.), University of California, San Francisco, San Francisco, California.

This work was supported by the National Center for Advancing Translational Sciences, National Institutes of Health, through UCSF-CTSI Grant Number B27552C. In addition, the research reported in this publication was supported by the National Institutes of Health under award number R21 DC016087-01A1.

The content of this article is solely the responsibility of the authors and does not necessarily represent the official views of the National Institutes of Health.

Paper previously presented, in part, at: Annual Meeting of the American Academy of Otolaryngology-Head and Neck Surgery Foundation, September 18-21, 2016; San Diego, California; and another portion of these data were presented at: Annual Meeting of the Society of Neurolnterventional Surgery, July 25-29, 2016; Boston, Massachusetts. suicidal ideations because of their tinnitus. ${ }^{2-9}$ A subset of tinnitus is rhythmic, termed "pulsatile tinnitus" (PT). PT accounts for up to $10 \%$ of patients with tinnitus. ${ }^{10-12}$ Some causes of PT are associated with a very high risk of intracranial hemorrhage, stroke, or blindness. Even the more benign causes of PT have a very high association with debilitating comorbid psychiatric disease. Some causes of PT can be treated. In our experience, treating an underlying cause of PT not only alleviates the risks of hemorrhage, stroke, or blindness but can also mitigate the comorbid psychiatric disease. Thus, identifying and treating causes of PT can be very beneficial to patients.

PT may be related to abnormal flow in vascular structures near

Please address correspondence to Matthew Amans, MD, MSc, UCSF, Department of Radiology and Biomedical Imaging, 505 Parnassus Ave, Room L349, San Francisco, CA, 94143; e-mail: matthew.amans@ucsf.edu,

- Indicates open access to non-subscribers at www.ajnr.org

http://dx.doi.org/10.3174/ajnr.A5833 
the cochlea and is often pulse-synchronous. ${ }^{1,11}$ The vascular structures with aberrant flow can be either venous or arterial. Approximately $40 \%$ of PT etiologies are venous, approximately $35 \%$ are arterial, and in nearly $25 \%$, the cause of PT is never identified. ${ }^{13}$ One of the venous etiologies of PT is a sigmoid sinus diverticulum (SSD). SSD is an outpouching from the sigmoid dural venous sinus that is usually laterally or anteriorly oriented and is often associated with an upstream transverse or sigmoid sinus stenosis. ${ }^{1,13-16}$ Altering the flow of venous blood through the SSD and the parent sinus by manual compression of the ipsilateral internal jugular vein is known to mitigate symptoms. $\mathrm{Pa}-$ tients report an increase in symptoms in high-flow states such as contralateral internal jugular vein compression, after exercise, or during pregnancy. ${ }^{13,17}$ Treatment of the offending SSD, which has been reported with both endovascular and open surgical techniques, can resolve PT. ${ }^{17-22}$

However, the exact pattern of blood flow in patients with SSD has not been studied. Two recent approaches provide the ability to explore the flow patterns in complex vascular geometries. One is direct measurement of the velocity field using MR imaging techniques; the other is using a numeric simulation approach to compute the velocity field. Advances in MR imaging have facilitated the visualization of the $3 \mathrm{D}$ velocity field of blood flow through the cardiac cycle. We have adapted these techniques for imaging the flow patterns in the transverse sinus, sigmoid sinus, and the internal jugular vein near to and including the jugular bulb (a region of venous sinus anatomy we will refer to as the SSIJ) using 3T MR imaging. ${ }^{23-25}$ Alternatively, with known boundary conditions (ie, the luminal surface and the inlet flow waveform), numeric simulations (computational fluid dynamics [CFD]) can be used to calculate the velocity field. The aim of this study was to apply these techniques to subjects with SSD and evaluate the blood flow patterns in this venous anomaly. We hypothesize that subjects with PT with SSD demonstrate a distinct flow pattern in the SSIJ and SSD that is absent in control subjects.

\section{MATERIALS AND METHODS}

We prospectively performed MR imaging analysis on adult patients with suspected venous etiology of pulsatile tinnitus and control subjects without pulsatile tinnitus using a study protocol approved by our institutional review board. All patients signed written informed consent to participate in this study.

\section{Patient Selection}

Patients were recruited from the University of California, San Francisco (UCSF) Pulsatile Tinnitus Clinic. The UCSF Pulsatile Tinnitus Clinic is a multispecialty clinic that evaluates patients with PT. PT was suspected to be of venous etiology if the patient described a low-pitched, pulse-synchronous PT that improved with ipsilateral neck compression and potential arterial (or other) etiologies (such as carotid atherosclerosis, fibromuscular dysplasia, tumor, or dural arteriovenous fistula) were not identified on prior imaging studies. All patients underwent the UCSF Pulsatile Tinnitus Clinic MR imaging and MRA protocol, which included brain MR imaging with fat-saturated postcontrast sequences, time-of-flight MRA of the head, time-resolved contrast-enhanced MRA of the neck through the circle of Willis, and postcontrast spoiled gradientrecalled imaging to evaluate venous sinus anatomy.

\section{Imaging}

The imaging protocol used in this study is an adaptation of contrast-enhanced MRA and 4D flow (4DF) described in prior articles. ${ }^{23-25}$ High-resolution contrast-enhanced MRA was performed on a 3T Magnetom Skyra scanner (Siemens, Erlangen, Germany). A 3D time-resolved imaging with a stochastic trajectory timing run with a $2-\mathrm{mL}$ gadolinium bolus was used to determine the venous phase of contrast opacification as the point when the contrast first appears in the distal transverse sinus. The full contrast-enhanced MRA study was then performed with a $20-\mathrm{mL}$ injection of gadolinium-based contrast agent at $2 \mathrm{~mL} / \mathrm{s}$ with an acquisition time of 35 seconds. Images had $0.7-\mathrm{mm}$ isotropic resolution covering an FOV of $280 \times 180 \mathrm{~mm}$ with an 84 -mm-thick slab. Image acquisition used an elliptic centric $k$-space ordering with the center of $k$-space synchronized to the venous phase as determined from the timing run. Following acquisition of the MRA, a finger-pulse-triggered 4D MR velocimetry study (4DF) was acquired at a $1.3-\mathrm{mm}$ isotropic resolution and $32-\mathrm{mm}$ slab coverage. Subsequently, 2D phase contrast was acquired in a slice transverse to the vessel in the mid-transverse sinus and proximal to any stenosis if one was present. Phase contrast in-plane resolution was $0.65 \times 065 \mathrm{~mm}$.

\section{Postprocessing}

The contrast-enhanced MRA vein and 4D MR velocimetry datasets were segmented using Clem-volume, an in-house software package. The velocity field, streamlines, path lines, vorticity, and helicity maps were generated from these data using Paraview (Kitware, Clifton Park, New York). In addition, a numeric simulation of the velocity field (ie, a CFD analysis) was performed using the contrast-enhanced MRA for the luminal surface boundary conditions and the inlet flow condition as acquired from the $2 \mathrm{D}$ phase contrast study. Several assumptions were made in our CFD analysis, including Newtonian flow, steady flow conditions, pressure of $0 \mathrm{~cm} \mathrm{H}_{2} \mathrm{O}$ at the outlet of the modeled segment, and rigid walls, as per our previously published method. ${ }^{24}$ To investigate the effects of an interventional treatment on the velocity field, we altered the geometry to provide a simulated, smooth, and continuous luminal surface that excluded the SSD. Path lines and streamlines were generated from the CFD-predicted velocity field.

\section{Flow Pattern Analysis}

The streamlines were extracted and visualized in Paraview, using seed points dispersed throughout the flow domain. As can be seen in the figures, regions of recirculating or vortical flow were identified by visual inspection.

\section{RESULTS}

Twenty-five patients with a suspected venous etiology of PT and 10 control subjects without PT were imaged using our protocol. Of the 25, five (20\%) had an SSD identified on MRA (Table). Streamlines of the CFD- and 4DF-resolved velocity field images for the 5 patients with SSD and 1 control subject are shown in 
Anatomic characteristics and treatments performed for the identified sigmoid sinus diverticula

\begin{tabular}{lcccccc}
\hline Subject & $\begin{array}{c}\text { SSD } \\
\text { Dimensions } \\
(\mathbf{m m})\end{array}$ & $\begin{array}{c}\text { Neck } \\
\text { Maximum } \\
\text { Width }(\mathbf{m m})\end{array}$ & $\begin{array}{c}\text { Orientation } \\
\text { of Long } \\
\text { Axis of SSD }\end{array}$ & Laterality & $\begin{array}{c}\text { Associated } \\
\text { Upstream } \\
\text { Stenosis }\end{array}$ & Treatment \\
\hline 1 & $6.0 \times 2.8 \times 3.2$ & 3.2 & Lateral & Left & Yes & None \\
& $6.8 \times 8.0 \times 4.2$ & 3.4 & Lateral & Right & Yes & Coiled \\
2 & $7.4 \times 5.5 \times 6.3$ & 5.1 & Anterior & Right & Yes & Surgical resurfacing \\
3 & $8.8 \times 5.7 \times 8.4$ & 6.4 & Anterior & Right & Yes & None \\
4 & $8.3 \times 3.9 \times 6.9$ & 6.6 & Lateral & Right & Yes & None \\
5 & $6.8 \times 7.5 \times 5.6$ & 4.1 & Lateral & Left & Yes & Stent \\
\hline
\end{tabular}

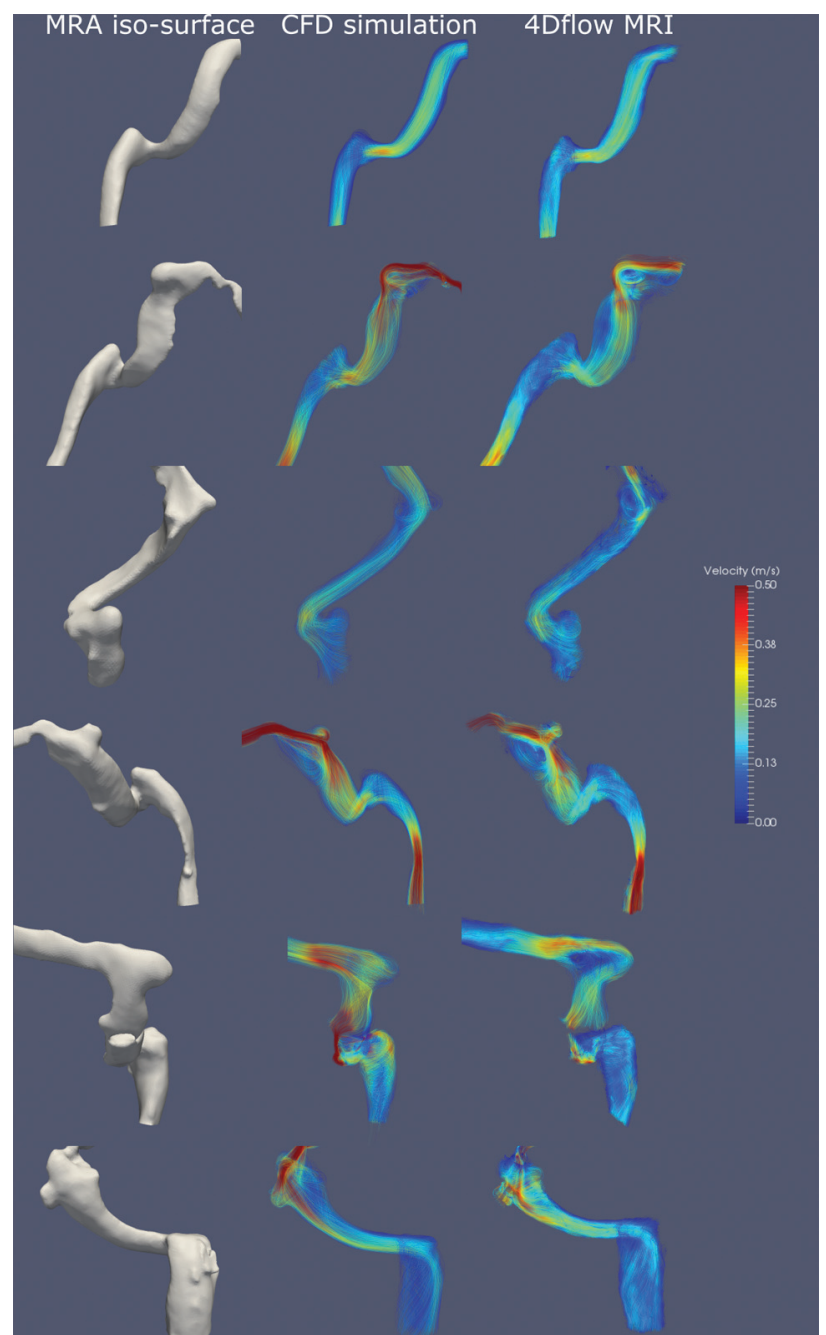

FIG 1. MRA isosurface MIP rendering, CFD-predicted flow fields, and 4D-flow MR imaging from 6 subjects. The first subject is a control subject. The other 5 are subjects clinically suspected of having a venous cause for pulsatile tinnitus who were found to have a sigmoid sinus diverticulum on MRA. The following observations are made in symptomatic subjects with a sigmoid diverticulum: 1) high velocity flow jet in an upstream stenosis in the transverse sinus directed at the SSD opening; 2) a flow jet into the SSD along the long axis of the SSD, either anteriorly or laterally directed; 3) a vortex of flow in the SSD; and 4) a strong vortex component of flow in the sigmoid sinus from the SSD. In the control subject, none of these flow components were present.

Fig 1. All SSDs had a bulbous shape directed away from the flow in the upstream sinus, either laterally or anteriorly, and all were associated with an upstream stenosis with a flow jet directed toward the SSD opening.

\section{Observations of Blood Flow from 4DF (In Vivo Measurement)}

In vivo measurement of blood flow in the SSIJ revealed some commonalities among patients with SSD: 1) a high-velocity flow jet is demonstrated at the stenosis in the transverse sinus, directed distally at the SSD opening; 2) the flow jet into the SSD is oriented along the long axis of the SSD (either anteriorly or laterally directed); 3) there is a vortical flow in the SSD; 4) a large component of rotational flow is present in the sigmoid sinus downstream from the SSD; and 5) none of these flow features were seen in the SSIJ of control subjects.

\section{Observations from CFD Flow Analysis}

The flow patterns observed in the CFD analysis were very similar to those identified by $4 \mathrm{DF}$. The flow jets through the stenoses, the vortices of flow in the SSD, and the flow recirculation in the sigmoid sinuses were all comparable between modalities. While the gross flow features were similar, there were some minor differences in the velocity fields resolved by $4 \mathrm{DF}$ and CFD in certain locations.

\section{Observations from Post treatment}

CFD analysis was performed on the expected post treatment geometries lacking SSDs and demonstrated the absence of flow recirculation in the parent sinus. Subject 2 elected to have surgical resurfacing of her SSD, which resulted in resolution of her PT symptoms. This subject subsequently underwent repeat MR imaging evaluation using our protocol. No flow was identified at the site of the prior SSD, and recirculation was no longer present in the parent sinus. The pre- and post treatment $4 \mathrm{DF}$ and CFD flow fields based on the pre- and post treatment MRA are shown in Fig 2. The SSD was excluded from the flow domain to simulate resurfacing of the pretreatment sinus geometry, and CFD was performed. For this simulated post-treatment geometry, the flow jet through the stenosis remained unchanged but the vortices in the sigmoid sinus downstream from the diverticulum remained.

\section{DISCUSSION}

We present a case series of 5 subjects with suspected venous PT and SSD in whom we imaged the anatomy and blood flow of the venous anomaly as well as the parent sinus using contrast-enhanced MRA and 4DF, and we also performed CFD analysis. All SSDs had similar anatomic and flow imaging, consisting of a bulbous outpouching projecting anteriorly or laterally from the sigmoid sinus, with the long axis of the SSD in the direction of a flow jet through the upstream transverse sinus stenosis. This study was an observational analysis that provides an illustration of the ability of imaging and computational methods to visualize complex 


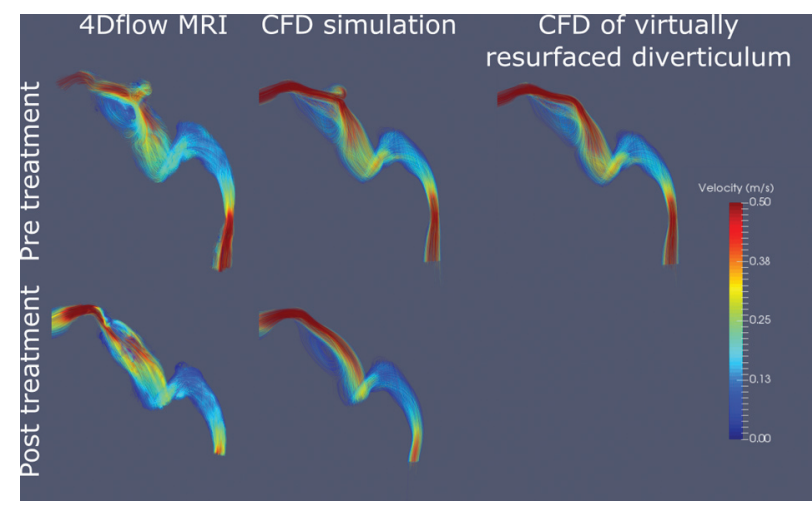

FIG 2. MRA, CFD simulation, and 4D-flow MR imaging from subject 2 before and after surgical exclusion of her sigmoid sinus diverticulum, which resulted in resolution of symptoms. There was an absence of flow in the SSD as well as lack of a vortex in the downstream sigmoid sinus. Only the SSD was excluded from the CFD flow domain. Thus, the flow jet through the proximal stenosis is unchanged.

flow patterns present in patients with venous diverticula. To have a clinical impact, inclusion of a broader range of geometrically varying SSDs is needed to provide a definitive link between geometry and flow and subsequently to the manifestation of PT symptoms.

While 4DF has been used fairly extensively in the vasculature, it has found the greatest application on the arterial side. To develop confidence in the $4 \mathrm{DF}$ measurement of the velocity field in the tortuous venous anatomy, we also performed numeric simulations of the flow fields using a CFD approach. Both 4DF and CFD demonstrated comparable flow patterns consisting of a flow jet through the stenosis, a vortex of flow in the SSD, and an additional vortex of flow in the parent sigmoid sinus. One case of repeat $4 \mathrm{DF}$ and $\mathrm{CFD}$ after SSD surgical exclusion, which resulted in cessation of PT, demonstrated an absence of flow in the SSD as well as resolution of the vortex of flow in the sigmoid sinus.

Previous publications have demonstrated the ability to evaluate flow in the SSIJ of patients with pulsatile tinnitus using both in vivo and patient-specific flow models, ${ }^{23}$ evaluate flow differences in the SSIJ of subjects with and without PT using CFD, ${ }^{24}$ and provide an overview of the varying flow patterns that can be seen in subjects with PT with different SSIJ anatomies using $4 \mathrm{DF}{ }^{25}$ Levitt et $\mathrm{al}^{26}$ used patient-specific MR imaging and catheter-obtained pressure measurements to generate CFD models of transverse sinus stenoses. The current work expands on prior articles by systematically reviewing a series of subjects with an anatomic aberration, SSD. In addition, we are the first group to evaluate flow in the dural venous sinuses of patients to establish the boundary conditions for the inlet flow conditions of our CFD analysis. This work also includes pre- and postsurgical evaluation of flow using both 4DF and CFD. In keeping with our group's prior publications of blood flow evaluation in the cerebral venous structures, we have again identified several interesting flow features in these patients.

The role that these flow patterns, specifically the vortices in the SSD and parent sinus, play in sound generation in patients with SSD and PT is unclear, and in fact, they may play no role at all. It is conceivable that turbulent flow downstream of the stenosis, within the SSD or at the outlet of the SSD, may contribute to sound generation. However, the unique pattern of flow presented in this work has not been previously demonstrated, to our knowledge. Our hope is to eventually use this technique to prospectively identify patients whose PT would be well-treated with surgical or endovascular exclusion of the SSD.

All subjects with SSDs had a concomitant transverse sinus stenosis. Transverse sinus stenosis has also been shown to be a cause of PT, and the high rate of comorbid sinus stenosis and SSD has been previously reported. ${ }^{18,20,22,27-32}$ The stenosis and subsequent flow jet may cause the SSD, similar to the effects of complex patterns of wall shear stress and spatial wall shear stress gradients previously reported in intracranial bifurcation aneurysms such as at the basilar tip. ${ }^{33,34}$ Our observation that the long axis of the SSD is coincident with the flow jet from the stenosis would seem to lend credence to this hypothesis; however, the relationship between the entities remains speculative.

To establish the role that geometric features of the diverticulum, such as maximal diameter and neck width, have in defining specific flow patterns such as vortices in the SSD and parent sinus, it will be necessary to expand this study to include a broader spectrum of geometric conditions. Expanding the study will similarly contribute to defining the link between flow patterns and sound generation in patients with SSD and PT. The unique pattern of flow presented in this work has not been previously demonstrated.

It is also unclear whether the stenosis, the SSD, or both are causing patients' symptoms. Many authors have treated both the stenosis and the diverticulum and reported resolution of symptoms ${ }^{27,31,32}$; others have treated only the SSD and also reported resolution of symptoms. ${ }^{18,22,28-30}$ We suspect that sound generation in these patients with PT is multifactorial, and the source of sound generation remains a focus of ongoing research.

All 5 patients with PT found to have an SSD were women. This may be simply a result of the small sample size, though there may be a component of idiopathic intracranial hypertension contributing to the transverse sinus stenosis in these patients, and idiopathic intracranial hypertension is more common in females. ${ }^{35} \mathrm{In}$ fact, subject 5 was found to have idiopathic intracranial hypertension based on standard criteria, ${ }^{36}$ and her symptoms greatly improved with stent placement in a transverse sinus stenosis, which decreased the pretreatment pressure gradient across the stenosis from 13 to $3 \mathrm{~mm} \mathrm{Hg}$.

There are several limitations to our study. Our small sample size limits the extent of the conclusions we can derive. Several assumptions were made in our CFD analysis, including Newtonian flow, steady flow conditions, zero pressure at the outflow, and a noncompliant vessel wall. While Newtonian flow is a reasonable assumption in capacious vessels such as the sigmoid sinus and jugular vein, the flow may be non-Newtonian in transversesigmoid junction stenosis. Steady flow conditions have been used in our prior work and have previously captured most flow information with a high degree of fidelity. ${ }^{24}$ Rigid wall conditions are likely a reasonable assumption in the transverse and sigmoid sinuses, which are encased by the thick dura and bone. However, the validity of this assumption is challenged by known changes in PT with lumbar puncture and removal of CSF, particularly in patients with idiopathic intracranial hypertension. ${ }^{37}$ In addition, 
the jugular vein has a very high capacitance and is known to not be a rigid structure. Our models in this work include only the jugular bulb that is, at least partly, bounded by the skull base.

\section{CONCLUSIONS}

This prospective case series applied $4 \mathrm{DF}$ and CFD analysis to patients suspected of having a venous etiology of PT and identified a consistent pattern of blood flow that was absent in controls. The flow pattern consists of a high-velocity jet through a transverse sinus stenosis directed into the SSD, vortical flow in the SSD, and a vortex of flow returning to the sigmoid sinus from the SSD. 4DF and CFD of the post treatment state show resolution of flow in the SSD and resolution of the vortex in the sigmoid sinus. The role the vortices play in sound generation for PT is unclear and remains a subject of future investigation.

Disclosures: Matthew R. Amans-RELATED: Grant: National Institutes of Health, Comments: This project was supported by the National Center for Advancing Translational Sciences, National Institutes of Health, through UCSF-CTSI Grant Number B27552C. In addition, the research reported in this publication was supported by the National Institutes of Health under award number R21 DC016087-01A1. Its content is solely the responsibility of the authors and does not necessarily represent the official views of the National Institutes of Health*; UNRELATED: Consultancy: Covidien, Comments: I have worked as a proctor for Pipeline embolization procedures. The total annual compensation is less than \$25,000. David Saloner-RELATED: Grant: National Institutes of Health*; UNRELATED: Grants/Grants Pending: National Institutes of Health, Comments: The National Institutes of Health is funding a grant related to this article. *Money paid to the institution.

\section{REFERENCES}

1. Krishnan A, Mattox DE, Fountain AJ, et al. CT arteriography and venography in pulsatile tinnitus: preliminary results. AJNR Am J Neuroradiol 2006;27:1635-38 Medline

2. Belli S, Belli H, Bahcebasi T, et al. Assessment of psychopathological aspects and psychiatric comorbidities in patients affected by tinnitus. Eur Arch Otorhinolaryngol 2008;265:279-85 CrossRef Medline

3. Weidt S, Delsignore A, Meyer M, et al. Which tinnitus-related characteristics affect current health-related quality of life and depression? A cross-sectional cohort study. Psychiatry Res 2016;237: 114-21 CrossRef Medline

4. Xu Y, Yao J, Zhang Z, et al. Association between sleep quality and psychiatric disorders in patients with subjective tinnitus in China. Eur Arch Otorhinolaryngol 2016;273:3063-72 CrossRef Medline

5. Pridmore S, Walter G, Friedland P. Tinnitus and suicide: recent cases on the public record give cause for reconsideration. Otolaryngol Head Neck Surg 2012;147:193-95 CrossRef Medline

6. Jacques D, Nozeret Y, Zdanowicz N, et al. Tinnitus and psychiatric comorbidities in liaison psychiatry analysis of three years in an audiophonology centre. Psychiatr Danub 2013;25(Suppl 2):S102-04 Medline

7. Zirke N, Seydel C, Arsoy D, et al. Analysis of mental disorders in tinnitus patients performed with Composite International Diagnostic Interview. Qual Life Res 2013;22:2095-104 CrossRef Medline

8. Seo JH, Kang JM, Hwang SH, et al. Relationship between tinnitus and suicidal behavior in Korean men and women: a cross-sectional study. Clin Otolaryngol 2016;41:222-27 CrossRef Medline

9. Seydel C, Haupt H, Szczepek AJ, et al. Three years later: report on the state of well-being of patients with chronic tinnitus who underwent modified tinnitus retraining therapy. Audiol Neurootol 2015;20: 26-38 CrossRef Medline

10. Liyanage SH, Singh A, Savundra P, et al. Pulsatile tinnitus. J Laryngol Otol 2006;120:93-97 Medline

11. Madani G, Connor SE. Imaging in pulsatile tinnitus. Clin Radiol 2009;64:319-28 CrossRef Medline

12. Harvey RS, Hertzano R, Kelman SE, et al. Pulse-synchronous tinnitus and sigmoid sinus wall anomalies: descriptive epidemiology and the idiopathic intracranial hypertension patient population. Otol Neurotol 2014;35:7-15 CrossRef Medline

13. Mattox DE, Hudgins P. Algorithm for evaluation of pulsatile tinnitus. Acta Otolaryngol 2008;128:427-31 CrossRef Medline

14. Weissman JL, Hirsch BE. Imaging of tinnitus: a review. Radiology 2000;216:342-49 CrossRef Medline

15. Sonmez G, Basekim CC, Ozturk E, et al. Imaging of pulsatile tinnitus: a review of 74 patients. Clin Imaging 2007;31:102-08 CrossRef Medline

16. Hou ZQ, Han DY. Pulsatile tinnitus in perimenopausal period. Acta Otolaryngol 2011;131:896-904 CrossRef Medline

17. Otto KJ, Hudgins PA, Abdelkafy W, et al. Sigmoid sinus diverticulum: a new surgical approach to the correction of pulsatile tinnitus. Otol Neurotol 2007;28:48-53 CrossRef Medline

18. Houdart E, Chapot R, Merland JJ. Aneurysm of a dural sigmoid sinus: a novel vascular cause of pulsatile tinnitus. Ann Neurol 2000; 48:669-71 CrossRef Medline

19. Eisenman DJ. Sinus wall reconstruction for sigmoid sinus diverticulum and dehiscence: a standardized surgical procedure for a range of radiographic findings. Otol Neurotol 2011;32:1116-19 CrossRef Medline

20. Liu Z, Chen C, Wang Z, et al. Sigmoid sinus diverticulum and pulsatile tinnitus: analysis of CT scans from 15 cases. Acta Radiol 2013; 54:812-16 CrossRef Medline

21. Santa Maria PL. Sigmoid sinus dehiscence resurfacing as treatment for pulsatile tinnitus. J Laryngol Otol 2013;127(Suppl 2):S57-S59 CrossRef Medline

22. Amans MR, Stout C, Dowd CF, et al. Resolution of pulsatile tinnitus after coil embolization of sigmoid sinus diverticulum. J Cerebrovasc Dis \& Stroke 2014;1:1010

23. Acevedo-Bolton G, Amans MR, Kefayati S, et al. Four-dimensional magnetic resonance velocimetry for complex flow in the jugular vein. Quant Imaging Med Surg 2015;5:635-37 CrossRef Medline

24. Kao E, Kefayati S, Amans MR, et al. Flow patterns in the jugular veins of pulsatile tinnitus patients. J Biomech 2017;52:61-67 CrossRef Medline

25. Kefayati S, Amans M, Faraji F, et al. The manifestation of vortical and secondary flow in the cerebral venous outflow tract: an in vivo MR velocimetry study. J Biomech 2017;50:180-87 CrossRef Medline

26. Levitt MR, McGah PM, Moon K, et al. Computational modeling of venous sinus stenosis in idiopathic intracranial hypertension. AJNR Am J Neuroradiol 2016;37:1876-82 CrossRef Medline

27. Zenteno M, Murillo-Bonilla L, Martínez S, et al. Endovascular treatment of a transverse-sigmoid sinus aneurysm presenting as pulsatile tinnitus: case report. J Neurosurg 2004;100:120-22 CrossRef Medline

28. Gard AP, Klopper HB, Thorell WE. Successful endovascular treatment of pulsatile tinnitus caused by a sigmoid sinus aneurysm: a case report and review of the literature. Intervent Neuroradiol 2009; 15:425-28 CrossRef Medline

29. Mehanna R, Shaltoni H, Morsi H, et al. Endovascular treatment of sigmoid sinus aneurysm presenting as devastating pulsatile tinnitus: a case report and review of literature. Intervent Neuroradiol 2010;16:451-54 CrossRef Medline

30. Park YH, Kwon HJ. Awake embolization of sigmoid sinus diverticulum causing pulsatile tinnitus: simultaneous confirmative diagnosis and treatment. Intervent Neuroradiol 2011;17:376-79 CrossRef Medline

31. Santos-Franco JA, Lee A, Nava-Salgado G, et al. Hybrid carotid stent for the management of a venous aneurysm of the sigmoid sinus treated by sole stenting. Vasc Endovascular Surg 2012;46:342-46 CrossRef Medline

32. Signorelli F, Mahla K, Turjman F. Endovascular treatment of two concomitant causes of pulsatile tinnitus: sigmoid sinus stenosis and ipsilateral jugular bulb diverticulum: case report and literature review. Acta Neurochir (Wien) 2012;154:89-92 CrossRef Medline

33. Meng H, Wang Z, Hoi Y, et al. Complex hemodynamics at the apex of an arterial bifurcation induces vascular remodeling re- 
sembling cerebral aneurysm initiation. Stroke 2007;38:1924-31 CrossRef Medline

34. Kulcsár Z, Ugron A, Marosfoi M, et al. Hemodynamics of cerebral aneurysm initiation: the role of wall shear stress and spatial wall shear stress gradient. AJNR Am J Neuroradiol 2011;32:587-94 CrossRef Medline

35. Satti SR, Leishangthem L, Chaudry MI. Meta-analysis of CSF diver- sion procedures and dural venous sinus stenting in the setting of medically refractory idiopathic intracranial hypertension. AJNR Am J Neuroradiol 2015;36:1899-904 CrossRef Medline

36. Friedman DI, Jacobson DM. Diagnostic criteria for idiopathic intracranial hypertension. Neurology 2002;59:1492-95 CrossRef Medline

37. Wall M. Idiopathic intracranial hypertension. Neurol Clin 2010;28: 593-617 CrossRef Medline 